\title{
Lattice Boltzmann simulation of rarefied gas flows in microchannels
}

\author{
Yonghao Zhang*, Rongshan Qin, and David R. Emerson \\ Department of Computational Science and Engineering, \\ Council for the Central Laboratory of Research Councils, \\ Daresbury Laboratory, Warrington, WA4 $4 A D, U K$
}

(Dated: January 25, 2006)

\begin{abstract}
For gas flows in microchannels, slip motion at the solid surface can occur even if the Mach number is negligibly small. Since the Knudsen number of the gas flow in a long microchannel can vary from 0 to 1.0 and the Navier-Stokes equations are not valid for Knudsen numbers beyond 0.1, an alternative method which can be applicable to the entire flow regimes is highly desirable. Lattice Boltzmann equation (LBE) approach has recently been expected to have such potential. However, some hurdles need to be overcome before it can be applied to simulate rarefied gas flows. The first major hurdle is to accurately model the gas molecule and wall surface interactions. In addition, the Knudsen number needs to be clearly defined in terms of LBE properties to ensure that the LBE simulation results can be checked against experimental measurements and other simulation results. In this paper, the Maxwellian scattering kernel is adopted to address the gas molecule and surface interactions with an accommodation coefficient (besides the Knudsen number) controlling the amount of slip motion. The Knudsen number is derived consistent with the macroscopic property based definition. The simulation results of the present LBE model are in quantitative agreement with the established theory in the slip flow regime. In the transition flow regime, it captures the Knudsen minimum phenomenon qualitatively. Therefore, the LBE can be a competitive method for simulation of rarefied gas flows in microdevices.
\end{abstract}

PACS numbers: 05.10.-a, 47.45.-n, 47.60.+i

\section{INTRODUCTION}

The technology associated with Micro-ElectroMechanical Systems (MEMS) or Micro-Total Analysis Systems ( $\mu$ TAS) has developed rapidly in the last decade and is set to revolutionize many important scientific areas. Of particular importance are chemical, biological and clinical analyses, where miniaturised systems offer the potential to significantly increase yields and reduce process time and reagent consumption. For gas flows in these devices, the quasi-equilibrium hypothesis, which leads to the Navier-Stokes equations, may be inappropriate. This is because the mean free path of the gas molecules may be comparable to the characteristic length scale of the microsystem. Recently, the LBE method has been developed as an alternative numerical scheme for fluid flow simulation [1-4], and it has also simulated gas flow in a microchannel $[5,6]$. The LBE method usually solves model Boltzmann equations such as Bhatnagar-Gross-Krook (BGK) model on a discrete lattice $[7,8]$. Its intrinsic kinetic nature makes it an attractive method for microfluidic flows where both microscopic and macroscopic behaviours are coupled.

Although gas flows in microsystems are usually creeping so that they are nearly incompressible, the Knudsen number can vary widely and readily exceed the NavierStokes equation limit of 0.1 . For practical applications, only a few macroscopic properties such as viscosity and

*Author to whom correspondence should be addressed. Email:Y.Zhang@dl.ac.uk flow rate are of interest which can determine the bulk motion characteristics. Therefore, Molecular Dynamics (MD), Direct Simulation Monte Carlo method (DSMC) and direct numerical simulation of the Boltzmann equation are too computationally expensive and impractical for applications where the microscopic details are not required. Significant effort has been made to improve and extend the validity of the Navier-Stokes equations beyond Knudsen numbers of 0.1 , or to construct complicated constitutive laws involving high order terms of Knudsen number which leads to Burnett-type equations [9]. The LBE method has the potential to improve this situation because it is efficient comparable to Navier-Stokes solvers and it can recover the Navier-Stokes equations. A preliminary link between the LBE and the Burnett-type equations has also been established [10]. In addition, the continuum, slip and transition flow regimes may exist together in microfluidic devices, e.g. a long microchannel. Hybrid algorithms that couple DSMC and NavierStokes methods have been tried to model these mixed flow regimes [11]. However, large errors can arise from inappropriate assumptions regarding, for example, the velocity distribution for gas molecules at the matching interface between two solutions [12]. Furthermore, these hybrid algorithms entail intensive computational effort for three-dimensional flow simulations. In principle, the LBE is valid throughout these mixed flow regimes and avoids any coupling problem. Consequently, the LBE may be a better method for gas flows in microdevices, particularly where mixed flow regimes are encountered.

He and Luo [13] and Abe [14] have demonstrated that the LBE can be derived from the Boltzmann equation. Shan and He [15] also show that the LBE is a special 
discrete velocity method of solving the Boltzmann equation. Although the above work did not produce new LBE models, theoretical connection between the LBE and the Boltzmann equation was established. Therefore, the LBE model can be valid for rarefied gas flows provided the Mach number is small. Moreover, since the standard BGK equation, a simplified model Boltzmann equation, is able to simulate highly nonequilibrium gas flows, the lattice BGK model should also be appilicable to rarefied microflows despite that the model is only validated for small Knudsen numbers. Numerical validation of LBE models against experimental data and well accepted DSMC results is needed for rarefied gas microflows in various geometries.

LBE methods have been extensively applied to simulate incompressible fluid flows with no-slip boundary condition at the wall. However, little has been done on the simulation of gas flows in microchannels, which are usually incompressible but tangentially slip at the wall. In order to tackle these flows, we need to model the gas molecule and solid surface interactions so that the slip motion at the wall boundary can be determined. In addition, the Knudsen number should be defined in terms of LBE properties that are consistent with the definition used in kinetic theory to ensure the comparability of the results obtained experimentally, theoretically and numerically.

\section{MATHEMATICAL MODEL}

For the sake of simplicity, the lattice BGK model is set as an example [8]:

$$
\begin{gathered}
f_{i}\left(x+\delta x_{i}, t+\delta t\right)-f_{i}(x, t)=-\frac{1}{\tau}\left[f_{i}(x, t)-f_{i}^{e q}(x, t)\right] \\
i=0,1, \ldots n
\end{gathered}
$$

where $f_{i}(x, t)$ is the density distribution function along the $i$ direction at the lattice site $x$ at time $t ; \delta x$ is the lattice length and $\delta t$ is the time step; $\tau$ is the dimensionless LBE relaxation time given by $\lambda / \delta t$ where $\lambda$ is the relaxation time; $f_{i}^{e q}$ is the local Maxwellian distribution function; the lattice velocity $c$, i.e. $\delta x / \delta t$, is chosen to obey mass, momentum and energy conservation. The density $\rho$ and bulk velocity $u$ can then be determined by $\sum_{i=0}^{n} f_{i}$ and $\sum_{i=0}^{n} f_{i} c_{i} / \rho$ respectively. For square lattice models, the LBE relaxation time $\tau$ can be related to the kinematic viscosity $\nu$ by $\frac{\delta x^{2}}{\delta t} \frac{(\tau-0.5)}{3}$. The factor -0.5 is the correction to make the LBE a second order method for solving incompressible flows [4].

In kinetic theory, the viscosity is linearly proportional to the mean free path, $l$, which is the mean distance a molecule travels between two consecutive collisions. As given by ref [16], $\nu=\frac{1}{2} \bar{c} l$ where the mean velocity of the molecule $\bar{c}$ is $\sqrt{\frac{8 k T}{\pi m}}$ ( $k$ is the Boltzmann constant, $T$ is the absolute temperature and $m$ is the molecular mass).
Therefore, Knudsen number can be expressed by

$$
K n=\frac{l}{H_{c h}}=\frac{2 \nu}{\bar{c} H_{c h}},
$$

where $H_{c h}$ is the channel height. Introducing a dimensionless channel height $H=H_{c h} / \delta x$, equation (2) becomes

$$
K n=\frac{2 \nu}{\bar{c} H \delta x}
$$

Since $\nu=\frac{\delta x^{2}}{\delta t} \frac{(\tau-0.5)}{3}$ and $c=\frac{\delta x}{\delta t}$, equation (3) becomes

$$
K n=\frac{2 c(\tau-0.5)}{3 \bar{c} H} .
$$

The lattice velocity $c$ depends on the lattice model, e.g. $c=\sqrt{\frac{3 k T}{m}}$ for the $\mathrm{d} 2 \mathrm{q} 9$ and $\mathrm{d} 3 \mathrm{q} 27$ models, $c=\sqrt{\frac{2 k T}{m}}$ for the $\mathrm{d} 2 \mathrm{q} 6$ model and $c=2 \sqrt{\frac{k T}{m}}$ for the $\mathrm{d} 2 \mathrm{q} 7$ model. Consequently, equation (4) could be further simplified, e.g. for the $\mathrm{d} 2 \mathrm{q} 9$ or $\mathrm{d} 3 \mathrm{q} 27$ lattice model:

$$
K n=\sqrt{\frac{\pi}{6}} \frac{(\tau-0.5)}{H} .
$$

Because the mean free path depends on microscopic details of molecular interaction, especially the collision frequency, the mean free path based Knudsen number could be different in various models despite the gases having the same macroscopic properties. In order to compare the results obtained by various models, we need to define the mean free path to be dependent only on the macroscopic properties, e.g. $l=\frac{\mu}{p} \sqrt{\frac{\pi}{2} \frac{k T}{m}}$, where $\mu$ is the dynamic shear viscosity and $p$ is the pressure. As a result, the Knudsen number in the standard BGK model given by equation (5) needs to be rescaled to

$$
K n=\sqrt{\frac{8}{3 \pi}} \frac{(\tau-0.5)}{H} .
$$

From equation(6), we can see the relation among $K n, \tau$, and $H$ can be exactly determined. Similarly, the Knudsen number for other lattice models can be obtained, for example, the Knudsen numbers for the d2q6 and $d 2 q 7$ LBE models can be given by

$$
K n=\left\{\begin{array}{lll}
\frac{4}{3 \sqrt{\pi}} \frac{(\tau-0.5)}{H} & : & d 2 q 6 \\
\frac{4 \sqrt{2}}{3 \sqrt{\pi}} \frac{(\tau-0.5)}{H} & : & d 2 q 7 .
\end{array}\right.
$$

Nie et al. [5] gave $K n=\frac{\alpha(\tau-0.5)}{H \rho}(\alpha$ is a constant to be determined numerically) and Lim et al. [6] asserted $K n=\frac{\tau}{H}$. In the present work, no free parameter is in the model to be tuned to produce desirable simulation results. Here, we need to emphasize that the Knudsen number differs by a constant factor among various lattice models. 


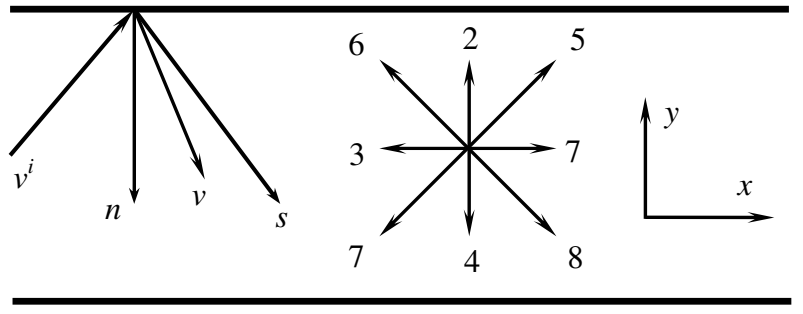

FIG. 1: Schematic diagram of gas surface interactions and velocity directions of a $2 \mathrm{D}(\mathrm{d} 2 \mathrm{q} 9)$ model in a microchannel, where $v^{i}$ is the incident velocity and $v$ is the reflected velocity of representative group of molecules. Here, $n$ is the normal direction and $s$ refers to the specular reflection direction.

The most important issue for extending LBE models to simulate rarefied flows is to model the boundary conditions at the walls capturing the underlying physics of gas molecule and surface interactions. The difference in boundary conditions will significantly affect the simulation results [17]. In order to solve the LBE, the distribution function of gas molecules $f^{+}$leaving the wall surface requires to be related to the incident molecules distribution function $f^{-}$. Generally, a scattering kernel is needed to determine $f^{+}$, and the details can be seen in refs $[18,19]$. The most widely applied kernel is the diffusive scattering model which can be interpreted as that the gas molecules lose all information of their state before collisions and are reflected obeying the Maxwellian distribution function. Recently, this diffusive scattering kernel has been implemented into the LBE model and the numerical results show good agreement with the analytical solution of the Boltzmann equation for Kramer's problem as the Knudsen number tends to zero [20]. However, for microflows, the Knudsen number can vary widely and gas surface interactions can be in-between diffusive and specular reflections. Therefore, more general boundary conditions are necessary for LBE models. Maxwell [21] expanded the diffusive kernel to a partly diffusive, $a$, and partly specular, (1- $a$ ) kernel. This accommodation coefficient $a$ is the most important parameter in describing solid surface interaction with the gas molecules. It is 1.0 for diffusive reflection and 0 for specular reflection. With the information of accommodation coefficient for various surface conditions available in the literature, we may establish a gas surface interaction model for the LBE method with practical implication. Since the Maxwellian scattering kernel has been applied and tested in solving Boltzmann equations, we propose to implement this gas surface interaction model and assess its effect on the simulation results.

A representative group of particles colliding with the wall is shown in Fig. 1, the post collision direction is usually in-between the normal direction $n$ and the specular reflection direction $s$, which could be characterized by the accommodation coefficient, $a$. If this Maxwellian kernel is to be implemented into a LBE (d2q9) model, the

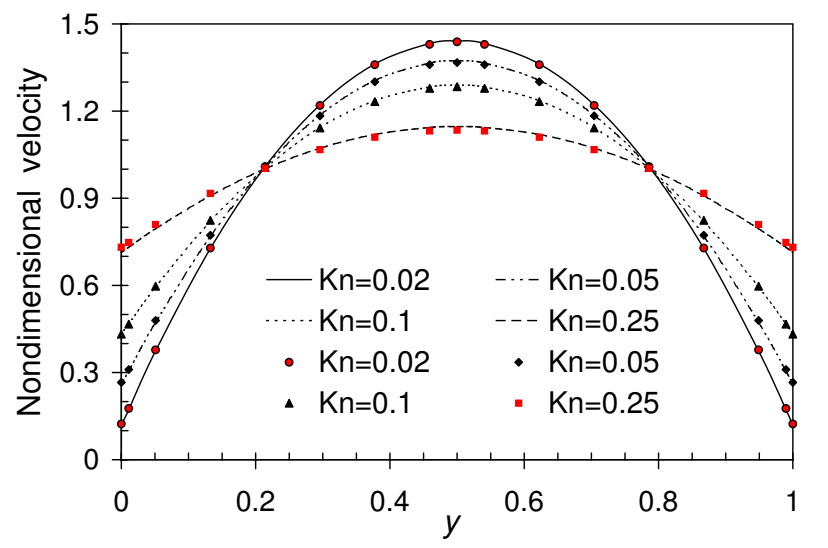

FIG. 2: Comparison of the velocity profiles of the fullydeveloped flows. The lines represent the results of Cercignani second order slip model while the symbols represent the simulation results of the present LBE model.

boundary condition at the upper wall can be expressed by

$$
\begin{aligned}
f_{8}(x, y, t+\delta t)= & (1-a) f_{5}(x-\delta x, y, t), \\
f_{7}(x, y, t+\delta t)= & (1-a) f_{6}(x+\delta x, y, t), \\
f_{4}(x, y, t+\delta t)= & a f_{5}(x-\delta x, y, t)+ \\
& a f_{6}(x+\delta x, y, t)+f_{2}(x, y, t) .
\end{aligned}
$$

This implementation of gas molecule and wall collisions is simple but captures the underlying physics, which is contrast to the empirical bounce back rule. It can be generalized to various geometric conditions and lattice models. A similar boundary condition could be obtained for the lower wall. Succi [17] has used a combination of the bounce back rule and specular reflection to generate slip effect at the wall. A reflection parameter is included which has recently been connected to the accommodation coefficient [22].

\section{RESULTS AND DISCUSSION}

Cercignani [23] used the BGK approximation and obtained a second order slip model for rarefied gas flows. As hard sphere gas has been generally accepted to be close to real gas flows in microsystems, Hadjiconstantinou [24] rescaled and improved the model for a hard sphere gas by considering Knudsen layers effects:

$$
\left.u\right|_{\text {wall }}=\left.1.1466 l \frac{\partial u}{\partial n}\right|_{\text {wall }}-\left.0.31 l^{2} \frac{\partial^{2} u}{\partial n^{2}}\right|_{\text {wall }},
$$

where $u$ is the slip velocity at the wall; $n$ is the normal direction to the wall; $l$ is the mean free path given by $\frac{\mu}{p} \sqrt{\frac{\pi}{2} \frac{k T}{m}}$; here diffusive reflection was assumed. In the literature, the value of the second order slip coefficient, which is 0.31 in equation (8), varies widely. For 


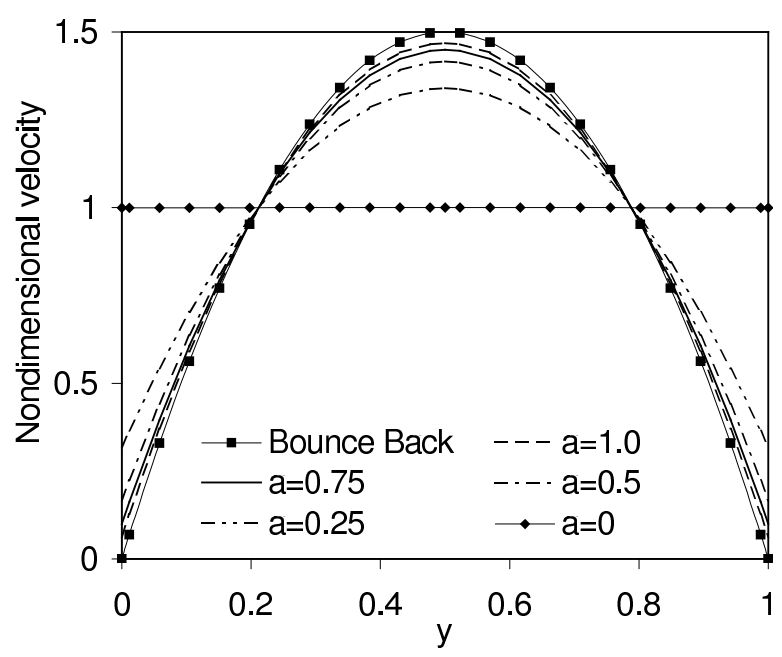

FIG. 3: The effect of the accommodation coefficient $a$ on the slip motion where the flow is fully-developed with $K n=0.01$.

example, it is 0.647 in ref [23]. In the flow regime with $K n<0.1$, where the Navier-Stokes equation is valid, the difference of the second slip coefficient has little effect on flow prediction. The comparison between the present LBE results and analytical solutions of the second order slip model is shown in Fig. 2, where the velocity is non-dimensionalized by the mean velocity. Excellent agreement has been achieved especially at small Knudsen number. The deviation starts at $K n=0.25$ where the validity of the Navier-Stokes equation with second order slip boundary condition is questionable. In the calculation, the gas flow is isothermal with Mach numbers well below 0.1. Tests have been carried out with the lattice number $H$ of 21,27 and 35 respectively and the numerical results are found $H$ independent. Along the channel, the lattice number is fixed at $50 \mathrm{H}$ and the pressure boundary condition is used.

For most LBE applications, the non-slip boundary condition is usually imposed by the so-called bounce back rule. When $K n$ increases, the bulk velocity is no longer zero at the wall and the empirical bounce back rule may not be valid. Lim et al. [6] used a specular reflection model and captured the slip motion of fluid at the wall for Knudsen numbers up to 0.155. However, a specular reflection indicates that there is no friction in the tangential direction, so that a plug-like bulk velocity in the microchannels will appear. At $K n=0.01$ where the slip starts, the impact of the accommodation coefficient on the velocity profile is shown in Fig. 3. If the bounce back collision is used, there is no slip motion at the wall while the velocity profile is plug-like as expected if a specular reflection model is assumed. With decreasing accommodation coefficient $a$, we can see the slip at wall is increasing. Therefore, an accurate determination of this coefficient is essential for LBE simulation results. Fortunately, this coefficient for many surface conditions is available in

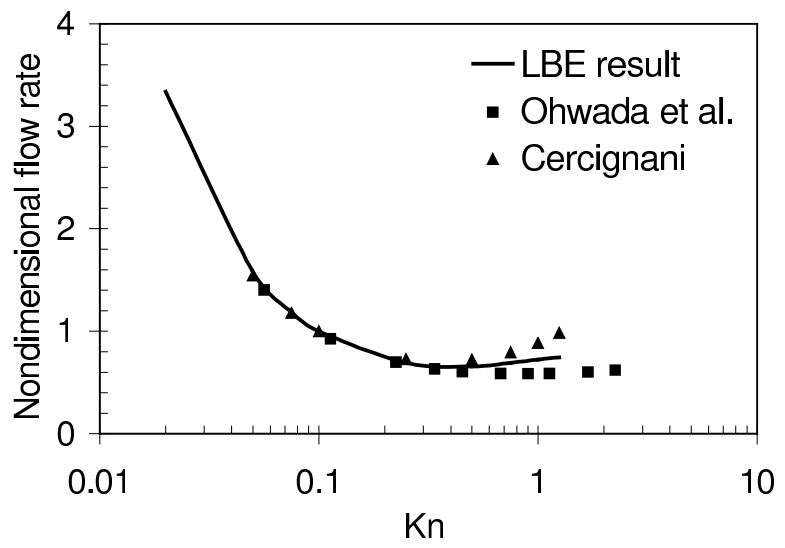

FIG. 4: Nondimensional flow rate as a function of the Knudsen number for fully developed flows. The data denoted by the solid squares were reported by Ohwada et al. [26]. The solid triangles represent the analytical solution of the NavierStokes equation with Cercignani's second order slip boundary condition [23].

the literature, hence the proposed gas-surface interaction model could promote the LBE model to be a design tool for microsystems.

By applying the present boundary condition, we can capture the famous Knudsen paradox (Knudsen minimum) phenomenon. Toschi and Succi [25] have recently independently reproduced the Knudsen paradox. Fig. 4 shows that the minimum mass flow rate occurs at $K n \approx$ 0.5 , where the flow rate is nondimensionalized by the flow rate at $K n=0.1$. Although the present LBE model prediction is more accurate than the Cercignani second order slip model at large Knudsen numbers, it starts to differ from the results reported by Ohwada et al. [26] at $K n \approx 0.4$. When $K n$ is large, equation (6) suggests that either the relaxation time $\tau$ is large or the channel height $H$ is small. In simulation, $H$ needs to be maintained at a reasonable value for the resolution, the only way to have a large $K n$ is to make $\tau$ large. Unfortunately, large $\tau$ will introduce significant numerical error, which needs to be tackled in order to extend the LBE model to simulate flows covering a broad range of Knudsen number.

In summary, a slip boundary condition has been proposed by adopting the Maxwellian scattering kernel to describe gas surface interactions. The accommodation coefficient has significant impact on the simulation results. The Knudsen number has been defined in terms of the LBE properties to be consistent with the macroscopic properties based definition commonly used in kinetic theory. This work has demonstrated that the LBE model is able to simulate isothermal gas flows in microchannels with large Knudsen numbers. In the slip flow regime, the present LBE model has achieved good agreement with the established analytical solutions, and it also captures the Knudsen minimum phenomenon qualitatively in the transition flow regime. Therefore, we may conclude that 
the LBE is a viable method to simulate rarefied gas flows in microsystems. Finally, we need to emphasize that the present work has only tackled isothermal rarefied gas flows in the simple geometry. Further work is required to extend current LBE models to be able to simulate rarefied thermal flows in complex geometries.

\section{ACKNOWLEDGEMENT}

This work is financially supported by UK Medical Research Council under grant reference 57719.
[1] G. McNamara and G. Zanetti, Phys. Rev. Lett. 61, 2332 (1988).

[2] F. J. Higuera and J. Jiménez, Europhys. Lett. 9, 663 (1989).

[3] F. J. Higuera, S. Succi, and R. Benzi, Europhys. Lett. 9, 345 (1989).

[4] Y. Qian, D. d'Humières, and P. Lallemand, Europhys. Lett. 21, 255 (1992).

[5] X. Nie, G. D. Doolen, and S. Chen, J. Stat. Phys. 107, 279 (2002).

[6] C. Y. Lim, C. Shu, X. D. Niu, and Y. T. Chew, Phys. Fluids 14, 2299 (2002).

[7] R. Benzi, S. Succi, and M. Vergassola, Phys. Rep. 222, 145 (1992).

[8] S. Chen and G. D. Doolen, Annu. Rev. Fluid Mech. 30, 329 (1998).

[9] D. A. Lockerby, J. M. Reese, D. R. Emerson, and R. W. Barber, Phys. Rev. E 70, 017303 (2004).

[10] Y. H. Qian and Y. Zhou, Phys. Rev. E 61, 2103 (2000).

[11] R. Roveda, D. B. Godstein, and P. L. Varghese, J. Spacecraft Rockets 37, 753 (2000)

[12] D. B. Hash and H. A. Hassan, Heat Transf. 10, 242 (1996).
[13] X. He and L.-S. Luo, Phys. Rev. E 55, R6333 (1997).

[14] T. Abe, J. Comp. Phys. 131, 241 (1997).

[15] X. Shan and X. He, Phys. Rev. Lett. 80, 65 (1998).

[16] S. Chapman and T. G. Cowling, The mathematical theory of non-uniform gases (Cambridge University press, Cambridge, 1970).

[17] S. Succi, Phys. Rev. Lett. 89, 064502 (2002).

[18] C. Cercignani, Theory and application of the Boltzmann Equation (Scotish Academic, Edinburgh, 1975).

[19] C. Cercignani, R. Illner, and M. Pulvirenti, The Mathematical Theory of Dilute Gases (Springer, New York, 1994).

[20] S. Ansumali and I. V. Karlin, Phys. Rev. E 66, 026311 (2002).

[21] J. C. Maxwell, Phil. Trans. R. Soc. 170, 231 (1879).

[22] M. Sbragaglia and S. Succi, nlin.CG/0410039 (2005).

[23] C. Cercignani, Institute of Engineering Research Report AS-64-19 (University of California, Berkeley, 1964).

[24] N. G. Hadjiconstantinou, Phys. Fluids 15, 2352 (2003).

[25] F. Toschi and S. Succi, Europhys. Lett. (in press).

[26] T. Ohwada, Y. Sone, and K. Aoki, Phys. Fluids A 1, 2041 (1989). 